\title{
Heat Consumption Technique of Cloud Data Center and Produce Distilled Water
}

\author{
Debabrata Sarddar, Gourab Dutta, Rajat Pandit
}

\begin{abstract}
Cloud computing is a recent and emerging technology of computer science and engineering. In cloud data center runs more than thousands of server and host runs for process and store the data. Due to this reason, the cloud data center produces a lot of carbon and heat which exhalation into the air thus the environment pollution occurs due to the cloud data center. That is why researches in energy consumption, saving energy, cooling renewable energy, environmental pollution control are a new researches area of cloud computing. In this research, we mainly concentrate on the heating problem of the cloud data center and produce distilled water by using the heat of the cloud data center. We transfer the heat from the cloud data center to the water tank through the channel and by using this heat we produce the distilled water. For produce, the distill water huge heat and cost required. In our research proposal for producing the distilled water, the main source of the heating energy is cloud data center so we can reduce the heating cost for producing the distilled water. By using this proposed model we not only reuse the heat of the data center but also we can control the heating problem of the cloud data center which is harmful to the environment.
\end{abstract}

Keywords: Cloud Computing, Energy Consumption, Environment Pollution, Waste Heat Reuse, Water Purification.

\section{INTRODUCTION}

In the mid 20th century, the cloud computing technology of computer science would change the world. Research is growing in a different field of cloud computing. Due to increasing the customer demand cloud data center size also increase. Cloud data center responsible for data storing, transmitting and processing all the information or data between the host. Cloud computing also provides the pay-as-you-go service for that reason the customer can use according to their demand i.e customer can use only the software or infrastructure or platform according to their needs and pay for that. For that reason, cloud computing is more popular to the customer although some security-related issue occurred due to all the data store into the third party vendor i.e who provide the cloud service although an agreement contract established between the cloud service provider and the cloud customer formally known as Service Level Agreement (SLA) [1]. For the data security of cloud computing, different kinds of data encryption techniques are used. Environmental pollution also occurs due to increasing the establishment of a new cloud data center to fulfill customer demand.

Revised Manuscript Received on December 30, 2019.

* Correspondence Author

Dr. Debabrata Sardar, Computer Science and Engineering, University of Kalyani, Kalyani, India.

Mr. Gourab Dutta*, Computer Science \& Engineering, Brainware Group of Institutions, Kolkata, India.

Mr. Rajat Pandit, Computer Science, West Bengal State University, Kolkata, India.

(C) The Authors. Published by Blue Eyes Intelligence Engineering and Sciences Publication (BEIESP). This is an open access article under the CC BY-NC-ND license (http://creativecommons.org/licenses/by-nc-nd/4.0/)
Lots of heat and carbon exhalation into the environment from a cloud data center and the climate of the environment also change due to heat and greenhouse effect.

Another and one of the major causes of the change of the climate is electricity because of many greenhouse gases like carbon dioxide and carbon monoxide emissions from the cloud data center during the electricity generation from cloud data center [2]. For cloud data center operators the huge amount of electricity required. In the year 2010 among the total world's electricity $1.5 \%$ has been consumed by the different data centers [3]. Due to the high power of the data center, the heating problem is one of the major problems. From the cloud data center, the heat and carbon emission to the environment and the temperature of the environment also increase. For the increasing, demand of the customer the cloud data center is constantly operated and it becomes over-provisioned in the working state, also for that reason a large amount of energy wastage [4]. We try to use the heating energy of cloud data center and by using this energy we produce the distilled water because for produce the distilled water huge energy required and these energies we got from cloud data center in this way we can reduce the heating problem of the cloud data center and also produce purified distilled water which is most required and essential thing of medical science. By using this proposed model we can control the heat of the cloud data center and we also reuse the heat for producing the distilled water. Distilled water is purified water and for producing the distilled water huge heat required the source of the heat is cloud data center. Distillation is a water purification technique by which we can produce purified water. Raw cool water boiled and produce the stream and then the stream hold into another chamber then this process is done periodically and removes the impurity of the water. To increase the purity of the water we use double still method. But we should collect the distilled water carefully and store it into the distilled chamber. We can remove a different type of contamination of the water at the boiling point of $100^{\circ} \mathrm{C}$. We can produce by using distillation method type II or type III water. For producing the distill water huge heat required for example $3 \mathrm{kw}$ electricity required to produce 4 liters distilled water but this huge heat we collect from the cloud data center and we use this heat to produce the distilled water.

In Section II, discussed the related work and in Section III discussed the proposed work and how to control the heating problem of the cloud data center and how to produce the distilled water by using the heat of the cloud data center. In the section, IV describes the basic block diagram of our proposed model. In section, $\mathrm{V}$ shows the flow chart of our proposed model and in section VI all about the conclusion and future activities.

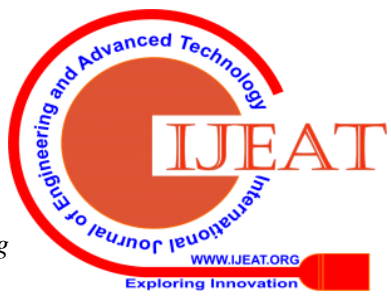




\section{Heat Consumption Technique of Cloud Data Center and Produce Distilled Water}

\section{RELATED WORK}

Several cooling techniques are used to scale down the heating temperature of the cloud data center. Some existing system uses a different technique like DVFS where the server turned on or off automatically depending on the workload of the data center. DVFS adjusts the voltage and energy depending on the workload of the server [5]. Smart cooling technology of the cloud data center also used with the concept of workload distribution in the different hard disks. Distributed metrology uses for sensing the pressure, temperature, and power of the cloud data center and try to coll the cloud data center depending on the sensor reading. The main disadvantages of this system are that it takes only the average reading of the sensor. But in case of emergency, it can not respond and another disadvantage of this system is operated by manpower [6]. By using different models and analysis tools [7] we can measure cloud data center performance and reading of energy saving during the run time of the data center.The Cloud data center provides a framework that provides the virtualization resource as a service. We can minimize the energy consumption of the cloud data center using a virtualization technique [8]. This technology supports the overcome the power from a different virtual machine on a single physical host and optimal utilization of the resource. In the potential use of cloud virtualization, we can reduce the energy expenditure of the cloud data center. Here the cloud data center utilizes less number of the server to offer the service of multiple devices [9]. Also, a new power-based scheduling algorithm [10] applies to the virtual machine for minimum power consumption of the virtual machine. In this method according to the customer requirement virtual machine dynamically shut down. By using this technique, we can scale down the energy and cost of the cloud data center. Due to the emission of carbon and heat from cloud data center environment pollution occur our main goal and objective reduce the heat emission from the cloud data center and make cloud computing technology like green cloud computing.

The green cloud data center mainly depends on three methods i.e the scheduling of use of renewable energy, dynamic power management and dynamic voltage frequency scaling [11]. For cooling the cloud data center chilled fluid passing through the pipes which placed nearer to the data center. By using this technique we can maintain the constant temperature inside the cloud data center. Immediate heating issues are a major problem. This technique can coll the cloud data center and kept a constant cooling system but it can not be applicable in case of overheating. For overheating another technology or by using manpower for coll the cloud data center. This technique monitor by the manpower for cooling the cloud data center [12]. In this cooling technique also required some additional energy of electricity for the chilled fluid. From one survey we see that in 2014 the power expenditure of cloud data center exceeded $42 \mathrm{TWh}$ and also we noticed that the co2 emission reach 670 million metric tons by the year 2020 [13]. For customer demand fulfillment, the cloud data center constantly operates in a working state, and huge energy is wastage [2]. For compressing the power expenditure of the cloud data center we use another method where the server goes to the low power mode or reduces the power during the ideal state of the server [14]. Greenhouse effect Also one of the major problems of the cloud data center. Due to the heating problem and carbon emission from the cloud data center the environment pollution occurred and the temperature of the environment also increases. For controlling the environment pollution different technologies are used in the cloud data center. Due to environmental pollution and global warming climate of the environment should be changed and this affecting the human lifestyle and health. By using 6R principles we can control the carbon emissions from the data center and also we can control the energy expenditure of the cloud data center. The 6R principles include revive, reuse, reduces, research, rescue and recycle [15]. Different kinds of green information and communications technology (ICT) and energy reduction techniques are mainly focused on environment pollution control due to the cloud data center. Also developed some energy consumption model, energy-aware cost, workload distribution into the cloud data center to gain an efficient trade-off between the cost of energy and data center performance. By using a measurable matrix we can calculate the ownership cost and total utilization cost of the cloud environment [16]. But the result only based on a single hardware component this does not applicable to the distributed system.

\section{PROPOSED WORK}

Cloud computing is a new and demanding technology in computer science and engineering. Nowadays cloud computing is a very useful and challenging area. Due to the heat emission from the cloud data center, the environment temperature increase and the greenhouse effect also occurred. All the previous work discusses energy consumption, load balancing, cooling technology, environmental pollution control, etc. All those previous work we follow that for coll the cloud data center extra energy required. To cool the data center and remove the heating problem all the previous techniques required an extra expense.

We proposed a new architectural model for the cloud data center where we extract the heat of the cloud data center and by using this heat we produce the distilled water. Heavy heat and energy of cloud data center wastage due to constantly service and fulfill the requirement of the customer by using the heat of cloud data center we produce distilled water. We keep a water chamber nearer to the data center and through the channel we transfer the heat from the cloud data center to the water chamber. The chamber contains raw normal water using the heat of the cloud data center to produce the stream from the water. Then through the channel, we collect the stream carefully from the water chamber to another storage block. By using this method we can remove the impurity of the water. To remove the impurity or for getting the better result we can do this procedure twice. Due to the heat of the cloud data center, the environment pollution occurs and the temperature of the environment also increases. In our proposed model the heat is not emissions to the air heat directly transfer to the normal raw water that's the way we can control the environment pollution and also control the temperature of the environment. In our proposed model did not require any kind of extra energy and heat for producing the distilled water whatever energy required we can transfer the heat from the data center to the water chamber. In this way, we can also reuse the heat of the cloud data center. 

for the medical industry and commercially also salvageable. For producing the distilled water huge energy or power required but in our proposed model did not require extra power or energy for producing the distilled water. In this way, we can control the heating problem of the cloud data center and without any extra energy, we can produce distilled water.

\section{BLOCK DIAGRAM OF OUR PROPOSED MODEL}

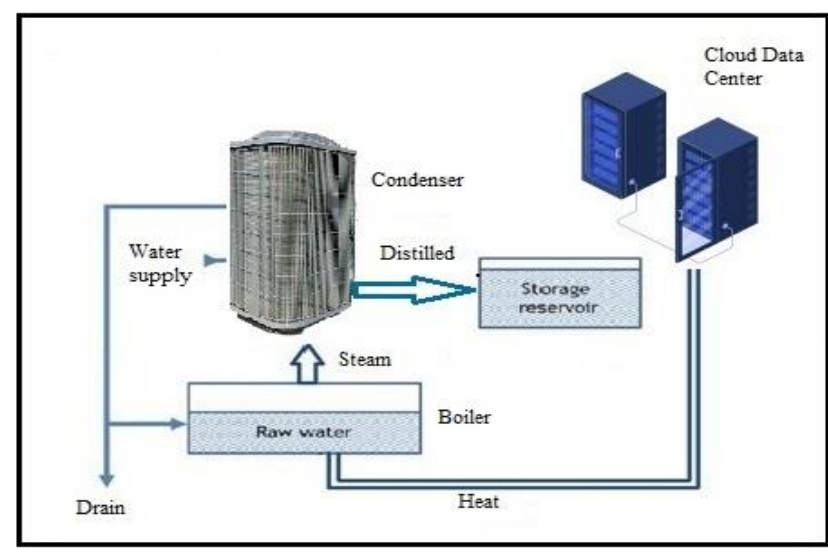

Fig. 1: Basic Block diagram

\section{FLOW CHART OF OUR PROPOSED MODEL}

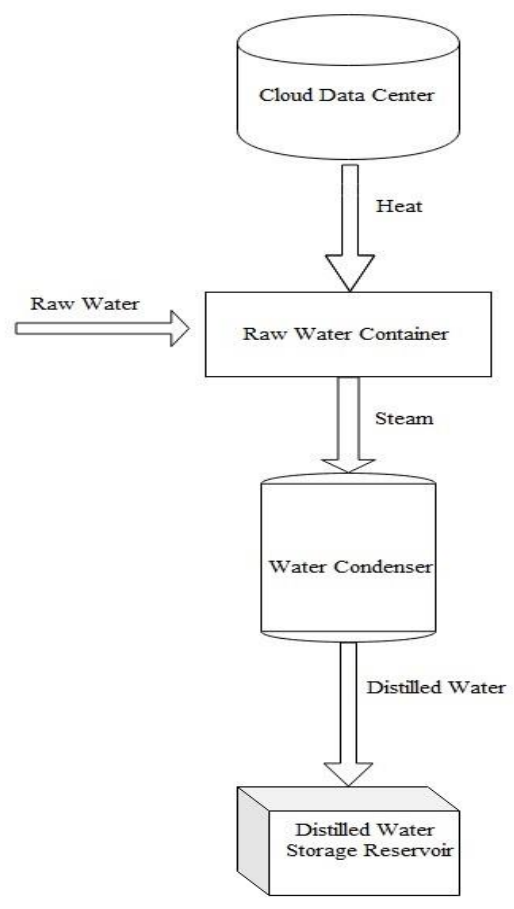

Fig. 2: Flow Chart

\section{CONCLUSION AND FUTURE WORK}

Nowadays a days environment pollution increases due to electricity generation from the cloud data center and greenhouse gas like hydrogen, carbon dioxide, carbon monoxide emission from the cloud data center. Due to the environment pollution temperature of the environment also increases and the climate of the environment also changes.
Distilled water is also the most useful and essential

Here, we mainly concentrate on the heating problem of the environment because of a cloud data center. We try to reduce the heating problem by cause of electricity generation against the cloud data center and by using this heat we produce distilled water. To produce the distilled water huge energy required and we reuse the heating energy from the cloud data center for producing the distilled water. By using our proposed model one side we can reduce the cost for producing the distilled water and another side we can control the heating problem of the cloud data center.

For fulfilling the demand of the customer constantly the huge energy wastage. In the future, we try to control the energy expenditure of the cloud data center by using different external techniques. Because huge energy expenditure is one of the major problems of the cloud data center.

\section{ACKNOWLEDGMENT}

We would like to convey our hearty appreciation and serious recognition to our respected research advisor Asst. Prof. Dr. Debabrata Sarddar for sparing his valuable time and assimilating new impeccable ideas at every phase of our research. Without his gentle cooperation, encouragement, and careful direction, we would not have been capable to accomplish this research work.

\section{REFERENCES}

1. R. Buyya, C. S. Yeo and Venugopal, "Market-oriented cloud computing: Vision, hype, and reality for delivering IT services as computing utilities" in Proc. HPCC2008, 2008, IEEE, pp 5-13.

2. S. Murugesan, "Harnessing Green IT: Principles and Practices, "Cutter Business \& mdash, IT Strategies Executive Report, IEEE Computer Society, Vol. 10, No. 1, 2007.

3. P. Corcoran, A. Andrae, "Emerging trends in electricity consumption for consumer ICT", Nat. Univ. Irelan, Galway, Ireland, Tech. Rep., 2013.

4. G. S. Akula and A. Potluri, "Heuristics for migration with consolidation of ensembles of Virtual Machines" in proc. COMSNETS, 2014, p. 1-4.

5. L. Shang, L.S. Peh, and N. K. Jha, "Dynamic voltage scaling with links for power optimization of Interconnection networks," In the 9th International Symposium on High-Performance Computer Architecture, pp. 91-102, February 08-12, 2003.

6. M. Alizadeh, A. Greenberg, D. A. Maltz, J. Padhye, P. Patel, B. Prabhakar, S. Sengupta, and M. Sridharan "Data Center TCP (DCTCP).” In Proc. SIGCOMM'10, Aug-Sep, 2010, New Delhi, India. pp. 63-74.

7. F. Chen, J. Schneider, Y. Yang, J. Grundy and Q. He, "An energy consumption model and analysis tool for Cloud computing environments" 1st ICSE Workshop on Green and Sustainable Software Engineering, June 2012, pp. 102- 107.

8. S. Roy and S. Gupta, "The Green Cloud Effective Framework: An Environment-Friendly Approach Reducing CO2 Level," in Proc. ICONCE 2014, pp. 233-236.

9. B. Yamini and D.V. Selvi, "Cloud virtualization: A potential way to reduce global warming," Recent Advances in Space Technology Services and Climate Change (RSTSCC), 2010, pp.55-57, 13-15 Nov. 2010.

10. A. J. Younge, G. Laszewski, L. Wang, S. L. Alarcon, and W. Carithers, "Efficient Resource Management for Cloud Computing Environments,' Proc. IEEE Int'l Green Computing Conf. (IGCC '10), pp. 357-364, Aug. 2010.

11. C. Gu, Z. Li, H. Huang, and X. Jia, "Energy Efficient Scheduling of Servers with Multi-Sleep Modes for Cloud Data Center", IEEE Trans. Cloud Comput., p. 1-1, 2018.

12. W. Daniel Hillis, Mark Duttweiler, Kenneth D. Salter, Randall A. Yates, "Balanced chilled fluid cooling system for a data center in a shipping container", U.S.patent, patent number: US 7,511,960 B2, March 31, 2009.

13. S. F. Smith, "Is Scheduling a Solved Problem?", in Multidisciplinary Scheduling: Theory and Applications, Springer, Boston, MA, 2005, p. 3-17.

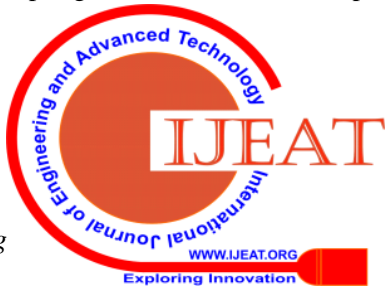




\section{Heat Consumption Technique of Cloud Data Center and Produce Distilled Water}

14. S. Agarwal, A. Datta, and A. Nath, "Impact of green computing in IT industry to make an eco-friendly environment," Journal of Global Research in Computer Science, Vol. 5, No. 4, pp. 5-10, Apr. 2014.

15. D. H. Liang, D. S. Liang, and C. P. Chang, "Cloud computing and green management", Intelligent System Design and Engineering Application(ISDEA), Second International Conference, 2012.

16. X. Li, Y. Li, T. Liu, J. Qiu, and F. Wang, "The method and tool of cost analysis for cloud computing," in the IEEE International Conference on Cloud Computing (CLOUD 2009), Bangalore, India, 2009, pp. 93-100.

17. D. D. Chaudhary, S. P. Nayse, L. M. Waghmare, "Application of wireless sensor network for greenhouse parameter in precision agriculture", International Journal of Wireless \& Mobile Networks, Vol. 3, No. 1, PP: 140-149, Feb. 2011.

18. R. Belsare, K. Deshmukh, M. Patil, Prof. Hattarge A.M., "Smart Greenhouse Automation", International Journal of Computer Science \& Engineering Technology, Vol. 5, No. 12, PP. 1127-1129, Dec. 2014.

19. D. Sarddar, G. Dutta , R. Pandit, P. Sen, “A Proposed Model to Control the Environmental Pollution due to Carbon Emission from Cloud Data Center", International Journal of Research in Electronics and Computer Engineering, Vol. 7, ISSUE 1, PP. 71-74, Jan - March. 2019.

20. K. V, G. N. Kodandaramaiah, "Cloud IoT Based Greenhouse Monitoring System", Keerthi.v Int. Journal of Engineering Research and Applications, Vol. 5, No. 10, pp. 35-41, Oct. 2015.

21. S. D. Bhagwat, A. I. Hulloli, S. B. Patil, A. A. Khan, A. S. Kamble, "Smart Greenhouse using IoT and Cloud Computing", International Research Journal of Engineering and Technology, Vol. 05, No. 03, PP. 2330 - 2333, Mar. 2018.

22. M. Soliman, T. Abiodun, T. Hamouda, J. Zhou, C. H. Lung, "Smart Home: Integrating Internet of Things with Web Services and Cloud Computing", In Cloud Computing Technology and Science (CloudCom), 2013 IEEE 5th International Conference, VoL 2, pages 317-320, Dec. 2013.

23. A. Uchechukwu, K. Li, Y. Shen, "Energy Consumption in Cloud Computing Data Centers", International Journal of Cloud Computing and Services Science, Vol. 3, No. 3, PP. 31-48, June 2014.

24. J.-Y. Son, et al, "Resource-aware smart home management system by constructing resource relation graph," IEEE Trans. On Consumer Electronics, vol. 57, No. 3, pp. 1112-1119, Aug. 2011.

25. J. Koomey, "Estimating Total Power Consumption by Server in the U.S and the World", Lawrence Berkeley National Laboratory, Stanford University, pp. 1-31, 2007.

26. P. Kogge, "The Tops in Flops", pp. 49-54, IEEE Spectrum, Feb. 2011.

27. J. Toress, "Green Computing, The next wave in computing", Jordi Torres, In Ed. UPC Technical University of Catalonia, Barcelona, 2010.

\section{AUTHORS PROFILE}

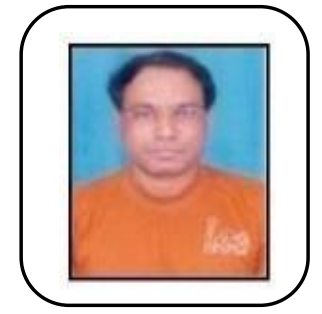

Dr. Debabrata Sarddar presently working as an Assistant Professor in the Department of Computer Science and Engineering at the University of Kalyani, Nadia, West Bengal, India. He completed his Ph.D. from Jadavpur University, Kolkata and he also completed his M.Tech in Computer Science \& Engineering in 2006 from DAVV, Indore. He also completed the B.E degree in Computer Science \& Engineering from the National Institute of Technology (NIT), Durgapur, West Bengal in 2001. He has published more than 200 research papers in various conferences and journals. His research area is Cloud Computing, Mobile System and Wireless Sensor Network.

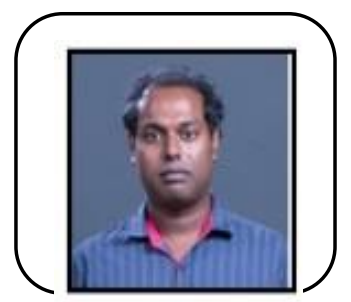

Mr. Gourab Dutta presently working as an Assistant Professor in the Department of Computer Science and Engineering at Brainware Group of Institutions - SDET, Kolkata. He completed his M.Tech in Computer Science \& Engineering from the Institute of Engineering and Management, Kolkata in 2013 and he also completed his B.Tech degree in Computer Science \& Engineering from Narula Institute of Technology, Kolkata in 2011. His main research work focuses on Cloud Computing, Bio-informatics. His total teaching experience is 6 years.

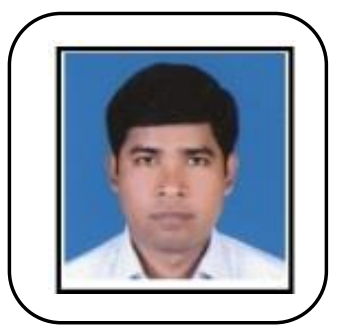

Mr. Rajat Panditpresently working as an Assistant Professor in the Department of Computer Science at West Bengal State University, West Bengal, Kolkata. He has completed his M.Tech in Information Technology in 2009 from West Bengal University of Technology, West Bengal, India. Also, he has completed his MCA in 2001 from Jadavpur University, Kolkata. His Wireless Sensor Network, and Mobile Computing. 